Note

Rev. bras. oceanogr., 50(único):59-62. 2002

\title{
Estudo da morfodinâmica da Barra da Icapara - SP com apoio de imagens obtidas por meio de sensoriamento remoto orbital
}

\author{
(Morphodynamic analysis of Barra de Icapara, SP with images obtained \\ by orbital remote sensing system)
}

\author{
Fernando Shinji Kawakubo' \& Ailton Luchiari ${ }^{2}$ \\ Faculdade de Filosofia, Letras, Ciências Humanas da Universidade de São Paulo \\ Laboratório de Aerofotogeografia e Sensoriamento Remoto (LASERE) \\ Departamento de Geografia
'Aluno de mestrado no programa de Geografia Física. fsk@usp.br
${ }^{2}$ Professor Doutor do Departamento. aluchiar@usp.br

- Descriptors: Coastal processes, Ilha Comprida, Barra de Icapara, LANDSAT 5, Digital processing, Multitemporal color compositions.

- Descritores: Processos costeiros, Illa Comprida, Barra de Icapara, LANDSAT 5, Processamento digital, Composições coloridas multitemporais.

\section{Introdução}

Uma das maiores dificuldades impostas ao estudo dos processos físicos e dinâmicos atuantes na zona costeira é a ausência de medições sistemáticas a respeito de ondas e marés ao longo do litoral brasileiro. Estes dados, segundo Muehe (1998), são praticamente restritos à localização de portos, não havendo portanto um recobrimento homogêneo ao longo do litoral.

A ausência destas medições tem dificultado o estabelecimento de modelos de circulação litorânea para diferentes segmentos da costa brasileira. Uma de suas consequiências é a dificuldade de se obter informações sobre as movimentações de sedimentos ao longo de sua linha de costa (Tessler, 1988). A análise comparativa de fotografias aéreas e imagens de satélite geradas em datas diferentes são

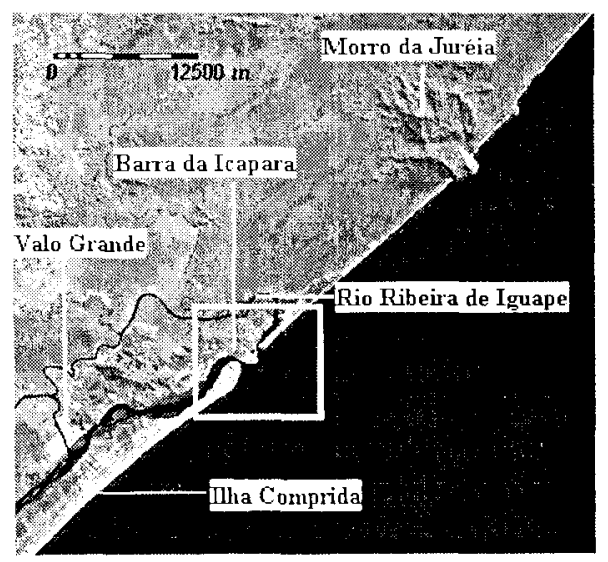

importantes fontes de informação para esse tipo de estudo.

A metodologia aplicada neste trabalho faz uso das técnicas de composições coloridas multitemporais geradas com imagens do satélite LANDSAT 5. O objetivo foi o de realizar uma análise da potencialidade destes dados no estudo relacionado a mudanças da linha de costa na Barra de Icapara, extremidade norte da Ilha Comprida.

A área de estudo está localizada entre as coordenadas $24^{\circ} 30^{\prime}-24^{\circ} 45^{\prime}$ de latitude Sul e $47^{\circ} 11^{\prime}$ - 47030' de longitude Oeste, na região do Vale do Ribeira - litoral Sul do Estado de São Paulo. Segundo a classificação de Silveira [(1964), apud Muehe (1998)], a região localiza-se no macro compartimento do litoral das planícies costeiras e estuarinas. A Figura l mostra em destaque a área de estudo e a sua localização em relação ao estado de São Paulo.

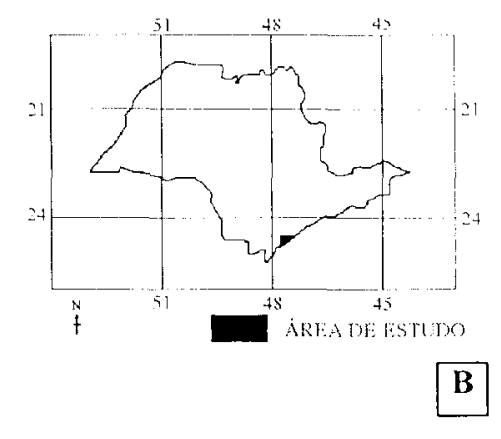

l:ig. 1. Banda TM5 do satélite LANDSAT 5 (A). A figura (B) mostra a sua localização em relação ao Estado de São Paulo. 


\section{Procedimentos executados}

O processamento digital foi feito utilizando as imagens do satélite LANDSAT 5, bandas TM3, TM4 e TM5 de junho de 1993 e de agosto de 1999. Ambas as imagens correspondentes à orbita 219 . ponto 77 .

O procedimento inicial realizado sobre as imagens consistiu na aplicação da correção radiométrica e geométrica. A correção radiométrica foi realizada para atenuar os efeitos causados pelo espalhamento atmosférico. O método utilizado foi o da simples subtração do pixel escuro, conforme descrito por Chavez (1988). A correção geométrica foi feita por meio de pontos de controle identificados na imagem e na carta digitalizada. Em seguida, as imagens foram reamostradas pelo método do vizinho mais próximo.

De modo a facilitar a interpretação dos dados, após as imagens terem sido pré-processadas, elas foram realçadas pelo aumento linear de contraste e pelas técnicas de composição colorida.

Para verificar as mudanças ocorridas na ponta Norte da I tha Comprida entre os anos de $1993 \mathrm{e}$ 1999, foram aplicadas sobre o conjunto de bandas a metodologia descrita por Eyton (1983) e Florenzano et al.(1988). Neste método é apresentado o uso de imagens de diferentes datas para a construção de composições coloridas, onde as mudanças ao longo do tempo são exibidas em diferentes cores e tonalidades.

De acordo com Eyton (1983), as mudanças que são ressaltadas na composição são resultantes da diferença na resposta espectral. Assim, alvos que aparecem com tonalidade escura em uma determinada época podem aparecer com tonalidade clara numa outra época. Esta mudança de reflectância pode ser ressaltada numa composição colorida multitemporal.
Neste método, as composições coloridas são obtidas por meio da superposição de três bandas iguais de duas ou três datas diferentes. As mudanças são apresentadas com cores primárias ou subtrativas, e nas áreas em que não houve mudanças, em tonalidades neutras, variando a sua intensidade do preto (para feições de baixa ou nula reflectância) ao branco (feições de alta reflectância).

\section{Resultados e discussão}

Nas três composições utilizadas, a cor vermetha foi associada às bandas do ano de $1999 \mathrm{e} \mathrm{a}$ cor ciano, às bandas do ano de 1993 (sobreposição do verde com o azul). A Figura 2 mostra a construção de uma composição utilizando este método.

Os resultados das composições mostraram que diferentes informações podem ser extraidas de cada banda. Essas informações devem, por sua vez, ser correlacionadas com outras fontes de informações para melhor compreender a complexidade dos processos atuantes.

A Figura 3A corresponde a composição utilizando a TM3. O resultado desta composição mostra que a vegetação apareceu em tonalidade escura e as estradas e linha de praia em branco. As diferenças mais expressivas observadas estão relacionadas à carga de sedimentos em suspensão na foz do rio Ribeira de Iguape.

A composição utilizando a TM4 resultou numa imagem com forte reflexão da energia pela vegetação. Devido às diferenças de iluminação entre as imagens, conforme mostra a Figura $3 \mathrm{~B}$, a vegetação apareceu com uma intensidade de vermelho claro. Nesta composição é possivel observar a presença dos sedimentos transportados ao longo da linha de costa e os processos erosivos e deposicionais que estão ocorrendo na região.

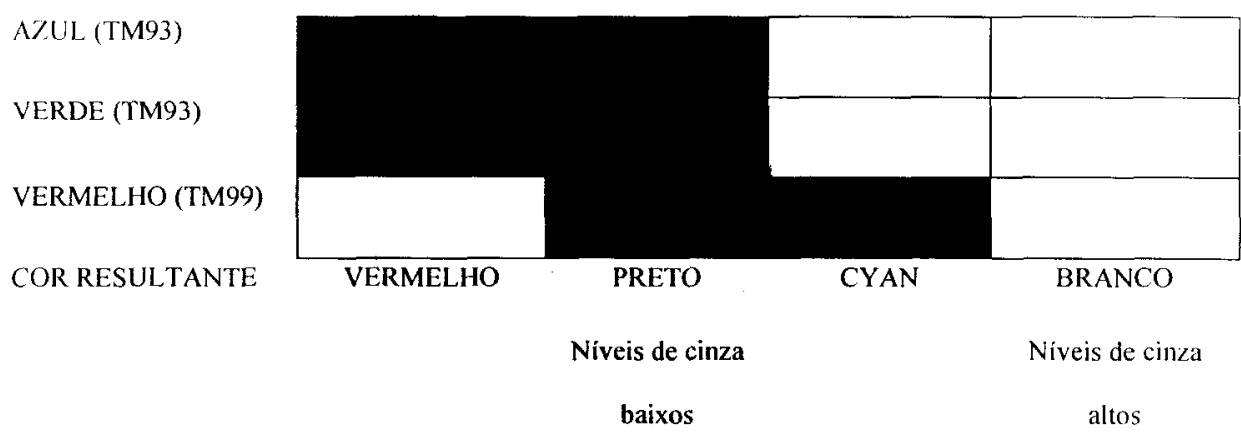

Fig. 2. Representação da construção de uma composição colorida multitemporal (adaptado de Eyton (1983) e Florenzano et al. (1988)). 

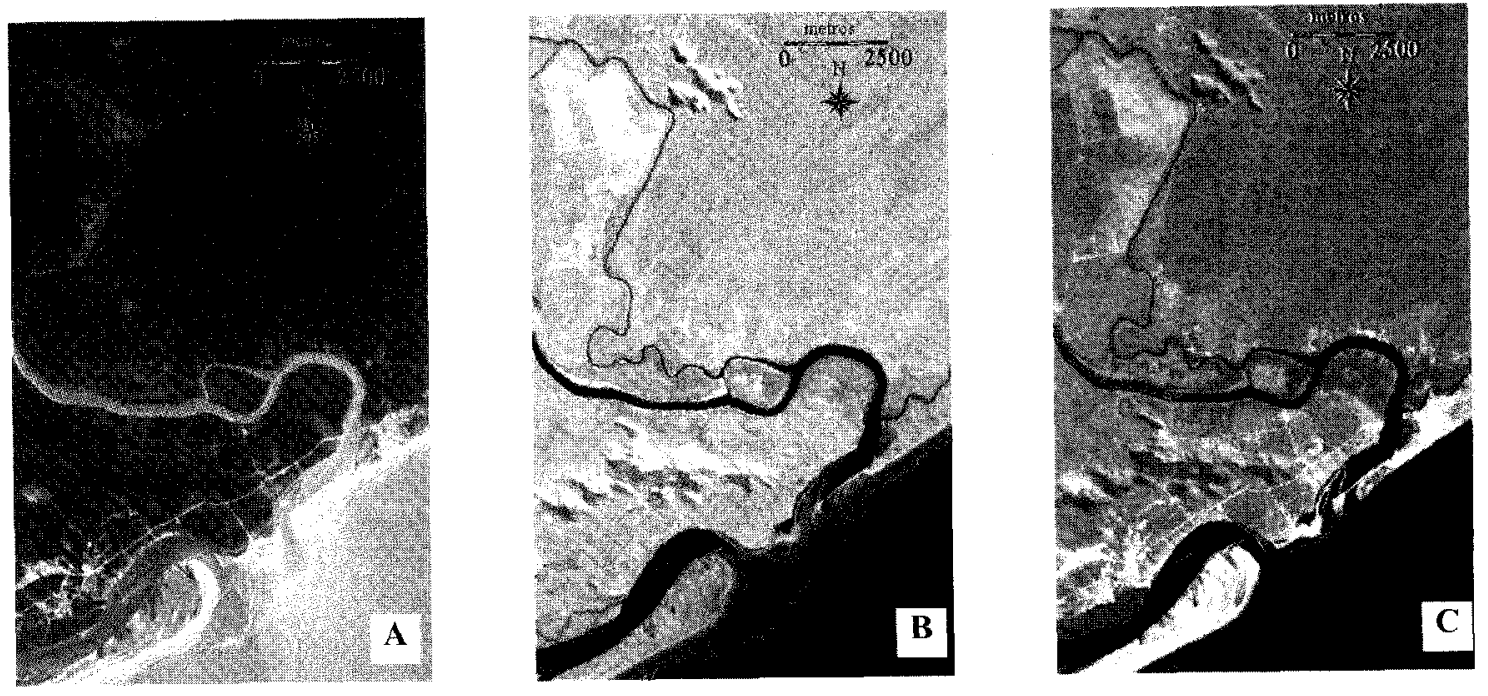

Fig. 3. A) Composição utilizando a TM3; B) utilizando a TM4; C) utilizando a TM5.

A superposição temporal da banda 5 constituiu numa imagem em que a vegetação possui baixa reflectância contrastando com as áreas de solo exposto, que possui maior reflexão. Esta composição foi a que forneceu o melhor resultado na identificação dos processos geomorfológicos e sedimentares, conforme pode ser observado na Figura 3C. A figura mostra o prolongamento da Ilha Comprida em direção $\mathrm{NE}$ e $\mathrm{N}$ realçada pela cor vermelha. As áreas que estão ocorrendo erosão pela cor ciano. Mostra também, nitidamente, na desembocadura do rio Ribeira de Iguape, o desenvolvimento de um esporão arenoso (cor vermelha) no sentido contrário ao crescimento da Ilha Comprida - SW.

Estudos realizados pela Geobrás S/A (1966) indicam que entre os anos de 1882 a 1965 a Ilha Comprida apresentou em sua direção predominante um crescimento de aproximadamente 2800 metros. No entanto este crescimento se fez de modo irregular, havendo um deslocamento médio para NE de 35 metros por ano.

Analisando a composição utilizando a TM5, pode-se constatar neste trabalho que entre os anos de 1993 e 1999, a extremidade Norte da Ilha Comprida teve um aumento de 220 metros, com média de crescimento de 37 metros/ano.

O recuo da Ilha de Iguape provocada pelos processos erosivos em suas margens foi considerada pela Geobrás S/A (1966) como sendo mais regular que o da Ilha Comprida. Em sua direção predominante a Ilha de Iguape deslocou cerca de 2800 metros. $O$ deslocamento médio anual na direção que corresponde ao maior recuo foi de 32 metros por ano.

O crescimento da Ilha Comprida está relacionado ao deslocamento predominante da corrente de vazante que atua no Mar Pequeno. Esta corrente de direção NE, ao interagir com as correntes de deriva litorânea formada por ondas provenientes do quadrante S-SE (corrente de mesmo sentido), resulta tanto no crescimento da ilha como na erosão da Ilha de Iguape (Tessler \& Mahiques, 1993).

Os sedimentos erodidos são transportados em direção ao oceano, que, posteriormente, são retidos pela ação das ondas na região da desembocadura lagunar, criando um conjunto de bancos arenosos submersos. Esses sedimentos, por sua vez, não são deslocados pelas correntes de deriva litorânea em virtude da ação do bloqueio fluvial e lagunar (Tessler, 1988), que além de reter o deslocamento do sedimento no sentido NE interfere nas características hidrodinâmica locais.

Um outro bloqueio que ocorre na foz do rio Ribeira está associado à carga de sedimentos transportados pela correntes de deriva litorânea geradas por incidência de ondas do quadrante NE-E. Ao sofrer o embate das correntes do sistema de ondas de S-SE atuante, proporciona o desenvolvimento do esporão arenoso presente. A direção de seu crescimento coincide com a direção das correntes geradas por ondas do quadrante NE-E, ou seja, SW.

\section{Considerações finais}

O método da composição colorida multitemporal mostrou-se eficiente na identificação das mudanças da linha de costa ocorridas na extremidade norte da Ilha Comprida. A composição utilizando a TM3 mostrou-se sensível para o estudo de carga de sedimentos transportados pelo rio Ribeira de Iguape. A composição utilizando a TM4 realçou as áreas em que ocorrem os processos erosivos e deposicionais além de informações a respeito de 
áreas em que ocorrem os processos erosivos e deposicionais além de informações a respeito de sedimentos transportados ao longo da linha de costa. A composição utilizando a TM5 foi a que forneceu o melhor resultado, destacando nitidamente os processos morfodinâmicos atuantes na região.

\section{Agradecimentos}

Estes autores agradecem ao apoio financeiro concedido pela FAPESP (processo 00/01477-5) e ao Laboratório de Aerofotogeografia e Sensoriamento Remoto (Lasere) do Departamento de Geografia da USP. Agradecimento à Rúbia G. Morato e especialmente ao Prof. Dr. Moysés G. Tessler pelos ensinamentos e admiração.

\section{Referências bibliográficas}

Chavez, P. S. 1988. An improved dark-objetct subtraction technique for atmospheric scattering correction of multispectral data. Remote Sens. Environ., 24:459-479.

Eyton, J. R. 1983. Landsat multitemporal color composites. Photogrammetric Engng. Rem. Sens, $2: 231-235$
Florenzano, T. G.; Pinto, S. A. F.; Filho, M. V.; Novo, E. L. M. \& Kux, H. J. H. 1988. Utilização de dados TM-LANDSAT para mapeamento de áreas submetidas a inundação na bacia do rio Panaíba. São José dos Campos, INPE-4570RPE/566. p. 1-50.

Geobrás S/A. 1966. Complexo Valo Grande, Mar Pequeno, Rio Ribeira de Iguape. São Paulo. Geobrás. 2v.

Muehe, D. 1998. O litoral brasileiro e sua compartimentação. In: Cunha, S. B. \& Guerra, A. J. T. eds Geomorfologia do Brasil. Rio de Janeiro, Bertrand do Brasil. p.273-349.

Tessler, M. G. 1988. Dinâmica sedimentar quaternária no litoral sul paulista. Phd Thesis. Universidade de São Paulo, Instituto de Geociências. 277p

Tessler, M. G. \& Mahiques, M. M. 1993. Utilization of coastal geomorphic features as indicators of longshore transport: examples of the southern coastal region of the State of São Paulo, Brasil. J. coast. Res., 9(3):823-830.

(Mamuscrito recebido 05 fevereiro 2002; revisado 02 julho 2002; aceito 11 março 2003) 


\title{
INSTITUTO OCEANOGRÁFICO DA UNIVERSIDADE DE SÃO PAULO CURSO DE PÓS-GRADUAÇÃO EM OCEANOGRAFIA
}

\author{
LISTA DAS DISSERTAÇÕES DE MESTRADO - LIST OF DISSERTATIONS (M.Sc.) \\ 2002
}

284. Aguiar, Valquiria Maria de Carvalho

- Estudo da dinâmica do fósforo no sistema estuarino de Santos/São Vicente em seu trajeto em direção à Baía de Santos. [Study of phosphorus dynamics in the estuarine system of Santos/São Vicente towards Santos'Bay].

285. Andrade, Lidiane Teixeira de Almeida

- Análise dos processos hidrodinâmicos e sedimentares da enseada da Fortaleza, Ubatuba/São Paulo. |Analyses of hydrodynamics and sedimentary processes of Fortaleza inlet, Ubatuba/SPI.

286. Araujo. Junior, Heitor Conrado de

- Variação sazonal do sulfeto de dimetila em águas superficiais do sistema estuarinolagunar de Cananéia. [Seasonal variations in the surface waters of the dimethilsulfide in the system estuarine-lagoon the Cananéia].

287. Arnalot. Elisabet Cornet

- Avaliação das concentrações de hidrocarbonetos do petróleo no Canal de São Sebastião, SP: influência do lançamento de um efluente petroquímico. [Evaluation of petroleum hydrocarbons in São Sebastião Channel, São Sebastião, SP: influence of the launching of a petrochemical effluentl.

288. Bertozzi, Carolina Pacheco

- Análise da pesca artesanal na região da Praia Grande (SP), no período 1999-2001. |Analysis of artisanal fishery in the Praia Grande (SP) region over the period 1999-2001|.

289. Bosquilha, Glaucia Elaine

- Estudo da distribuição de surfactantes aniônicos e de polifosfatos no sistema estuarino de Santos/São Vicente e Baía de Santos (São Paulo, Brasil) e avaliação das metodologias aplicadas. ISurfactants anionics and polyphosfates distribution in estuarys system of Santos/São Vicente and Santos'Bay (SP, Brazil) and application methodologys avaliation |. 
290. Calil, Paulo Henrique Rezende

- Dinâmica da interação de vórtices isolados com correntes de contorno oeste num oceano de 1,5 camadas. [Dynamics of the interaction of isolated eddies with western boundary currents in a 1.5 layer ocean $\mid$.

\section{Caballero, Natalia Venturmi}

- Estudo das características do ambiente de fundo e sua relação com a macrofauna bentônica, numa área adjacente a uma refinaria de petróleo: porção nordeste da Baía de Todos os Santos, Bahia, Brasil. IStudy of the bottom environment characteristics and their relationship with the benthic macrofauna, in an area adjacent to a petroleum refinery: northeast portion of Todos os Santos Bay, Bahia, Brazil].

\section{Castelão, Guilherme Pimenta}

- Um estudo sobre os fluxos de calor na superfície do Atlântico Tropical usando os dados do PIRATA. [A heat budget study in Tropical Atlantic using the PIRATA datal.

\section{Chen, Fernando Shimomura}

- Diagnóstico das condições oceanográficas na Bacia de Santos com base nos resultados da Comissão OCEANO SUDESTE-I do N/Oc. Antares. [Diagnostics of the oceanographic conditions in the Santos Bight based on the results of the OCEANO SUDESTE-I Comission of the N/Oc. Antares].

\section{D'Agostino, Luiz Fernando}

- Avaliação das variações paleoceanográficas do neoquaternário na margem continental sudeste do Brasil com base no estudo das associações de foraminíferos. [Evaluation of the neoquaternary paleoceanographic variations in the SE continental margin of Brazil based on the study of foraminifera associations].

\section{Fonseca, Carlos Augusto}

- Variabilidade da superfície do mar na região da retroflexão da corrente norte do Brasil com base em resultados de modelo numérico e dados altimétricos. |Variability of the sea surface height in the region of retroflection of the north Brazil Current based on results of numerical models and altimetric datal.

\section{Martins, César de Castro}

- Avaliação da introdução de esteróis fecais e hidrocarbonetos marcadores geoquímicos em sedimentos da Baía do Almirantado, Península Antártica. |Evaluation of fecal sterols and geochemical markers hydrocarbons in sediments from Admiralty Bay, Antarctic Peninsula]. 


\section{Navarro, Doris Graziela}

- Porichthys porosissimus (Teleostei: Batrachoididae): alimentação e atividade alimentar diária no litoral de Ubatuba, sudeste do Brasil. |Porichthys porosissimus (Teleostei: Batrachoididae): diet and diel feeding activity in south-western Atlantic, Brazil].

298. Oliveira e Azevedo, Gabriela Fernandes de

- Variabilidade sazonal (outono-primavera) da produtividade primária e biomassa fitoplanctônica na Enseada do Flamengo, Ubatuba, litoral norte do Estado de São Paulo. |Seasonal variability (autum-spring) of the primary production and phytoplankton biomass in the Flamengo Bay, Ubatuba, State of São Paulol.

299. Portugal, Ana Margarida Marques

- Manguezais de Guaratiba frente à perspectiva de elevação do nível médio relativo do mar, Baía de Sepetiba, Estado do Rio de Janeiro - Brasil. [Sea level rise scenarios for Guaratiba's mangroves, Sepetiba Bay, Rio de Janeiro State Brazill.

300. Vaz-dos-Santos, André Martins

- Idade e crescimento da merluza, Merluccius hubbsi Marini, 1933 (Teleostei: Merlucciidae) na zona econômica exclusiva entre o Cabo de São Tomé (RJ) e o Chui (RS), Brasil. [Age and growth of the common hake Merluccius hubbsi, Marini, 1933 (Teleostei: Merlucciidae) in the economic exclusive zone between Cabo de São Tomé (RJ) and Chuí (RS), Brazill.

\section{Yogui, Gilvan Takeshi}

- Ocorrência de compostos organoclorados (pesticidas e PCBs) em mamíferos marinhos da costa de São Paulo (Brasil) e da Ilha Rei George (Antártica). |Occurrence of organochlorine compounds (pesticides and PCBs) in marine mammals from São Paulo Coast (Brazil) and King George Island (Antarctica)].

\section{LISTA DAS TESES DE DOUTORADO - LIST OF PH.D. THESIS}

\section{2}

\section{Abessa, Denis Moledo de Souza}

- Avaliação da qualidade de sedimentos do sistema Estuarino de Santos, SP, Brasil. [Sediment quality assessment of the Santos Estuarine System, SP, Brazil].

\section{Attolini, Fabiano da Silva}

- Padrões de distribuição de poliquetas macrobentônicos na região de plataforma externa e talude superior entre Cabo Frio (RJ) e Cabo de Santa Marta (SC), costa sudeste do Brasil. [Distributional patterns of macrobenthic polychaeta in the outer shelf and upper slope between Cabo Frio (RJ) and Cabo de Santa Marta (SC), Southeast cost, Brazil]. 
- A evolução da pesca de linha-de-fundo e a dinâmica de população do peixe-batata, Lopholatilus villarii (Teleostei: Malacanthidae) na margem continental da costa brasileira entre os paralelos $22^{\circ}$ e $28^{\circ} \mathrm{S}$. [Evolution of bottom long-line fisheries and population dynamies of the tilefish, Lopholatilus villarii (Teleostei: Malacanthidae) in the continental margin along the Brazilian coast from Lat. $22^{\circ} \mathrm{S}$ to $28^{\circ} \mathrm{S}$.

\section{Coelho Filho, Petronio Alves}

- Crustáceos decápodos, isópodos e estomatópodos da plataforma continental externa e bancos oceânicos do Nordeste do Brasil (Programa REVIZEE NE III). [Crustacean decapodas, isopodas and stomatopodas from the outer continental shelf and oceanic banks of northeastern Brazil (REVIZEE Program NE-III)].

\section{Heitor, Simone Ribeiro}

- Composição e distribuição da macrofauna bentônica em áreas sob influência da disposição oceânica de esgotos municipais na Baixada Santista e no Canal de São Sebastião, São Paulo, Brasil. [Composition and distribution of the benthic macrofauna in areas with occurs the oceanic disposition of municipal sewage in the Baixada Santista and the São Sebastião Channel, São Paulo, Brazill.

\section{Luz, Betty Rose de Araíjo}

- Distribuição espacial da macrofauna bêntica nos recifes costeiros da Praia dos Carneiros (PE). [Spatial distribution of benthic macrofauna in the coastal reefs of Praia dos Carneiros (PE)|.

\section{Margariños, Leticia Burone}

- Foraminíferos bentônicos e parâmetros físico-químicos da Enseada de Ubatuba, São Paulo: estudo ecológico em uma área com poluição orgânica. [Benthic foraminiferans and physico-chaemical parameters of Enseada de Ubatuba, São Paulo: an ecological study from an organic polluted areal.

\section{Moser, Gleyci Aparecida Oliveira}

- Aspectos da eutrofização no sistema estuarino de Santos: distribuição espaçotemporal da biomassa e produtividade primária fitoplanctônica e transporte instantâneo de sal, clorofila- $a$, material em suspensão e nutrientes. [Santos estuarine system eutrophication aspects: phytoplanktonic biomass and primary productivity spatial-temporal distribution and instantaneous transport of salt, chlorophyll- $a$, suspended matter and nutrients].

125. Rachid, Bauer Rodoarte de Figueiredo

- Avaliação ecotoxicológica dos efluentes domésticos lançados pelos sistemas de disposição oceânica da Baixada Santista, SP. |Ecotoxicological evaluation of urban sewage discharged by oceanic disposal systems of Baixada Santista region|. 
126. Silva, Jodir Pereira da

- Estudo da biologia reprodutiva da Caratinga Eugerres brasilianus, (Pisces, Gerreidae) no complexo estuarino-lagunar de Cananéia-Iguape, SP. [Reproductive biology studies of Mojarra Eugerres brasilianus, (Pisces, Gerreidae) in the Estuarine Lagoon complex of Cananéia-Iguape, SPl.

127. Skowronski, Rodrigo Soares Pereira de

- Distribuição espacial e variação temporal da meiofauna, com ênfase para o grupo nematoda, na enseada Martel (Antártica). ISpatial and temporal variation of meiofauna, emphasizing Nematodes, at inlet Martel (Antaretica)]. 



\title{
INSTRUÇÕES AOS COLABORADORES DAS PUBLICAÇÕES DO INSTITUTO OCEANOGRÁFICO
}

\section{COMISSÃO EDITORIAL}

São publicações do Instituto Oceanográfico, a Revista Brasileira de Oceanografia (Brazilian Journal of Oceanography) e Relatórios Técnicos do Instituto Oceanográfico.

\section{REVISTA BRASILEIRA DE OCEANOGRAFIA (Brazilian Journal of Oceanography)}

\begin{abstract}
A Revista Brasileira de Oceanografia (Brazilian Journal of Oceanography) destina-se a divulgar textos de literatura cientifica no campo da Oceanografia, e demais ciências a ela relacionadas, classificados nas seguintes categorias: a) Trabalho original; b) Notas; c) Revisão de conjunto ou atualização.
\end{abstract}

\section{Natureza do texto}

a) Trabalho original - Um texto é considerado original, quando corresponde a uma significativa extensão ao conhecimento ou entendimento e é de tal forma, que permita a um pesquisador qualificado avaliar objetivamente as medidas e observações, repetir cálculos ou raciocínios teóricos, bem como julgar as descobertas e a precisão do trabalho realizado.

b) Notas - Corresponde a uma comunicação pouco extensa ou de natureza preliminar, embora a redação eventualmente não permita aos leitores avaliar informações como nas condições indicadas para o item a; ainda assim contribui para o avanço científico do campo considerado.

c) Revisão de conjunto ou atualização - Corresponde a uma avaliação geral de uma área do conhecimento, na qual informações já publicadas são reunidas, analisadas e discutidas. Autores de tais artigos deverão suplementar essas informações com contribuições pessoais, dar crédito a todos os trabalhos publicados que contribuíram para o desenvolvimento do assunto, incluindo os que não tenham recebido a adequada divulgação.

\section{NORMAS PARA APRESENTAÇÃO}

\section{Normas Gerais}

Os trabalhos devem ser inéditos e destinar-se exclusivamente à Revista Brasileira de Oceanografia (Brazilian Journal of Oceanography). Os trabalhos deverão ser redigidos em inglês. $O$ manuscrito se aceito para publicação, e o padrão lingüístico não for satisfatório, a Comissão Editorial solicitará ao autor para que o encaminhe para uma revisão por especialista qualificado.

Os originais dos trabalhos submetidos devem ser encaminhados em três vias, em espaço duplo em folhas tamanho A4, em uma só face, mantendo as margens laterais de $3 \mathrm{~cm}$ aproximadamente. Todas as páginas devem ser numeradas consecutivamente, em algarismos arábicos, em local definido e de maneira uniforme.

Os trabalhos destinados à categoria:

a) Trabalho original - 30 páginas, incluindo as ilustrações

b) Notas -7 páginas no máximo, incluindo as ilustrações.

c) Revisão de conjunto ou atualização - 50 páginas no máximo, incluindo ilustrações.

\section{Estrutura do trabalho original}

No preparo do trabalho original deverá ser observada, sempre que possivel, a estrutura convencional dos artigos científicos na seguinte ordem: título, autor(es), "abstract", resumo em português, descritores, introdução, material e métodos, resultados, discussão, agradecimentos e referências bibliográficas. 
Título - Deve ser breve e indicativo da exata finalidade do trabalho; no caso de ser necessário título longo, recorrer a subtítulo; versão do título para o inglês. Deve ser indicado o título corrente (running head).

Autores - Nome(s) do(s) autor(es), com a respectiva fillação cientifica (entidade à qual estão vinculados e endereço para correspondência).

Abstract - Em inglês, localizado entre o título e o texto, com no máximo 200 palavras, sem o emprego de parágrafos. Consiste em resumo breve do conteúdo do artigo: dever ser conciso e claro, ressaltando os resultados mais importantes.

Resumo - Em português, localizado após o Abstract, com no máximo 200 palavras, sem o emprego de parágrafos. Deve ser breve, conciso e claro, ressaltando os resultados mais importantes.

Descritores - O número de Descritores deve ser no máximo 8, cm português e inglês.

Introdução - Deve estabelecer com clareza o objetivo do trabalho, relacionando-o com outros do mesmo campo e apresentando de forma suscinta, a situação em que se encontra o problema investigado. Extensas revisões de literatura devem ser substituídas por referências aos trabalhos mais recentes, onde tais revisões tenham sido apresentadas.

Material e métodos - A descrição dos métodos usados deve limitar-se ao suficiente, para possibilitar a perfeita compreensão dos mesmos; processos e técnicas já descritos em outros trabalhos, devem ser referidos somente por citação, a menos que tenham sido consideravelmente modificados.

Resultados - Devem ser apresentados com clareza e sempre que necessário, acompanlados de tabelas e material ilustrativo adequados. Os dados numéricos devem ser apresentados em tabelas e figuras.

Discussão - Deve restringir-se à avaliação dos resultados obtidos e de suas possíveis causas e consequiências, relacionando as novas contribuições aos conhecimentos anteriores. Evitar hipóteses ou generalizações não baseadas nos resultados dos trabalhos.

Agradecimentos - Quando necessário, devem ser mencionados antes das referências bibliográficas.

Referências bibliográficas - Devem ser ordenadas alfabeticamente por sobrenome do autor, sendo consideradas apenas as referências mencionadas no texto. As referências devem ser completas, incluindo as iniciais dos nomes dos autores citados, ano de publicação, titulo completo do artigo, volume, fascículo e número de páginas (iniciais e finas). Títulos de periódicos devem ser abreviados de acordo com o "World list of science periodicals". Para citação de livros, deve ser mantida a sequiência: autor(es), ano de publicação, título completo do livro, edição (a partir da Segunda), local de publicação, casa publicadora (editora comercial), número total de páginas ou volume (se mais de uma na coleção). A seguir alguns exemplos:

\section{Artigos e periódicos:}

Gray, J. S. 1977. Animal-sediment relationships. Oceanogr. mar. Biol. a. Rev., 12:223-261.

\section{Livro:}

Orth, R. J. 1977. The importance of sediment stability in seagrass communities. In: Coull, B. C. ed. Ecology of marine benthos. Columbia, Univ. South Carolina Press. p. 281-300.

Zar, J. H. 1984. Biostatistical analysis. $2^{\text {nd }}$. Ed. Englewood Cliffs, N.J., Prentice IIall. $718 p$.

\section{Relatório:}

Sondotécnica/INPH. 1977. Comportamento hidráulico e sedimentológico do Estuário Santista. Relatório Técnico. $2 v$.

Tese:

Cavalcanti, L. B. 1976. Caracterização do Canal de Santa Cruz (Pernambuco) em função dos parâmetros físicoquimicos e pigmentos fotossintéticos. Tese de livre-docência. Universidade Federal de Pernambuco, Departamento de Oceanografia. $115 \mathrm{p}$.

Obs.: Os resumos apresentados em eventos não podem ser citados como referências bibliográficas. Caso necessário, deverão ser citados no rodapé do texto.

Ilustrações: Todo material ilustrativo (gráficos, fotografias, desenhos, mapas) deve ser designado no texto como Figuras. Devem Ter numeração seqüêncial, em algarismos arábicos. As legendas deverão ser datiloğrafadas em folha a parte. As ilustrações deverão ser identificadas, com a indicação do nome do autor e título abreviado do artigo. Os desenhos devem ser feitos em papel branco ou vegetal (sem dobras), com tinta nanquim ou utilizar impressora de boa qualidade. As letras e números das figuras devem ser suficientemente grandes para que permitam uma redução que não as tornem ilegiveis. O mesmo tipo de letra deve ser adotado para todas as figuras. As fotografias devem ser bem nítidas e contrastadas. Autores que incluirem ilustrações coloridas devem contactar a Secretária da Comissão Editorial. A impressão colorida será paga pelo autores. 
Tabclas - Devem ser numeradas consecutivamente, com algarismos arábicos, ter título e se necessário legenda, prescindindo do texto para a sua compreensão.

\section{Preparação eletrônica do texto}

Ao preparar um trabalho, a apresentação geral deverá ser a simples mais possível, evitando o uso das opções de justificação, hifenização, símbolos, etc., do processador de texto utilizado. Devem ser utilizados disquetes formatados por microcomputador IBM-PC ou compativel, no sistema MS-DOS 6.0.

As tabelas e figuras devem ser submetidas em arquivos diferentes daquele do texto, com nomes de fácil interpretação. No texto principal, deverá ser indicada, aproximadamente, a localização da cada figura ou tabela, pelo uso do nome do arquivo em disco e/ou legenda correspondente no manuscrito. As tabelas deverão ser editadas no mesmo processador usado no texto principal.

Processadores de textos - É aceito o seguinte processador de texto: Microsoft Word.

Formatos para figuras e fórmulas - Figuras e fórmulas eletronicamente submetidas deverão ser encaminhadas em arquivos separados no formato MS Windows. Na impossibilidade do uso deste, os arquivos deverão ser gravados na forma binária.

\section{Separatas}

Os autores receberão gratuitamente 50 separatas; separatas adicionais poderão ser solicitadas por ocasião da revisão da prova, a preço de tabela.

\section{NOTAS}

Os trabalhos destinados à categoria $\underline{\mathbf{b}}$ devem seguir estruturas:

Título e nome dos autores

Texto - Poderá incluir ilustrações e tabelas

Agradecimentos

Referências bibliográficas

\section{REVISÃO DE CONJUNTO OU ATUALIZAÇÃO}

Os trabalhos destinados à categoria $\mathrm{c}$ devem seguir a seguinte estrutura:

Título e nome dos autores

Abstract

Resumo

Texto - poderá incluir ilustrações e tabelas

Agradecimentos

Referências bibliográficas

\section{RELATÓRIOS TÉCNICOS DO INSTITUTO OCEANOGRÁFICO}

Os Relatórios Técnicos do Instituto Oceanográfico destinam-se a divulgar monografias sobre temas marinhos, pesquisas cientificas, registro de atividades de cruzeiros oceanográficos e registro de dados dos principais parâmetros oceanográficos e meteorológicos, decorrentes de observações realizadas nas Bases do Instituto Oceanográfico.

Os Relatórios Técnicos poderão apresentar a mesma estrutura do trabalho Original. No entanto, a critério dos autores, um ou mais itens da estrutura convencional, poderão ser dispensados.

Os trabalhos devem conter no máximo 50 páginas, incluindo as ilustrações. 


\section{INSTRUCTIONS FOR CONTRIBUTORS}

"Brazilian Journal of Oceanography" is a publication of the Instituto Oceanográfico da Universidade de São Paulo. The Brazilian Journal of Oceanography publishes scientific contributions on oceanography which include physical oceanography, marine chemistry, marine geology and geophysics, biologial oceanography, fishery oceanography and related fields. The contributions will be classified in one of the following categories: a) Article; b) Notes; c) Review.

a) Article - Original papers corresponding to the general scope of the journal.

b) Notes - Original contributions with limited scope or preliminary nature.

c) Review - A review paper of some specific field of oceanography, representing a general overview of the scientific contributions on the field.

\section{GENERAL INSTRUCTIONS}

Papers must be original and destined exclusively for the Brazilian Journal of Oceanography. Contributions to the regular issues should be in English. If the manuscript is accepted for publication, but the linguistic standard is not adequate, the Editorial Board will recommend to the author to submit it for grammatical review by a specialized reviewer.

\section{Manuscript submission}

The original paper must be submitted to the Editor of the journal in three copies of the text and figures. The text must be typed with double spacing on one side of white sheet with marginal space of about $3 \mathrm{~cm}$. All pages mut be sequentially numbered. The maximum pages of the manuscript for each category are:

a) Article - maximum 30 pages, including figures and tables.

b) Notes - maximum 7 pages, including figures and tables.

c) Review - maximum 50 pages, including figures and tables.

\section{Organization of the manuscript}

The desirable style of the organization of a paper is as follows:

Title - Must be short and indicative of the objective of the paper.

Author(s) - Full name(s) of the author(s) should be previded.

Affiliation(s) - The author(s) affiliations(s) and address for correspondence should be given.

Running title - Running head of title must be indicated not exceending 50 characters including space.

Abstract - The Article and Review papers must include a brief abstract which should not exceed 200 words.

Resumo - In Portuguese, located after Abstract, whith maximum 200 words whithout paragraphs.

Key words - The maximum of eight descriptive key words should be listed below the "Resumo".

Introduction

Material and methods

Result

Discussion

Acknowledgement

References - The reference list should be in alphabetical order according to the family name of the first author. A perfect correspondence should exist between citations in the text and the list provided in the Reference section. The references must be complete, including the family names of the authors cited, year of publication, complete title of the article, standard abbreviation of the journal title, volume, issue and page numbers (beginning and end). Journal title should be abbreviated according to the World list of science periodicals. For citation of books, the following order is recomended: author (or editor), year of puvblication, complete title, number of editions (if any), publication location, name of publisher and total number of pages. Follow the punctuation and style shown in the example below: 
Periodical journal:

Gray, J. S. 1977. Animal-sediment relationships. Oceanogr. mar. Biol. a. Rev., 12:223-261.

Book:

Orth, R. J. 1977. The importance of sediment stability in seagrass communities. In: Coull, B. C. ed. Ecology of marine benthos. Columbia, Univ. South Carolina Press. p. 281-300.

Zar, J. H. 1984. Biostatistical analysis. $2^{\text {nd }}$. Ed. Englewood Cliffs, N.J., Prentice Hall. 718 p.

Report:

Sondotécnica/INPH. 1977. Comportamento hidráulico e sedimentológico do Estuário Santista. Relatório Técnico. $2 v$.

Thesis:

Garfield III, N. 1990. The Brazil current at subtropical latitudes. Ph.D. Thesis. University of Rhode Island. $121 \mathrm{p}$.

Figures - All ilustrations (drawings, graphs or photo graphs) should be desiganated in thext as figures and be consecutively numbered with arabic numerals. Figure legends must be typed in separate sheets and all figures must be identified by name of author and number of the figure in a corner. Drawings should be made whith black India ink on bristol board. Figures made by computer using good quality printer are also accepted. Photographs must be clear and contrated. Authors to incluede colour plate in a paper should contact the Editor-Chief. The cost of colour work will be invoiced to authors.

Tables - Tables whith their heading on top should be consecutively numbered whit Arabic numerals and type on separate sheets. Headings should be brief and explanatory of the purpose of table.

\section{Preparation of the text with word processador}

When the manuscript is accepted for publication, the editor will ask the author a hard copy of the final version of the text, original figures and floppy disk $(3.5 \mathrm{or} \mathrm{cd})$. the text should be written by and word processador should be indicated. The standard ASCII format files are also accepted.

\section{Reprintes}

The author will receive 50 free reprints. When the author receives the gallery proofs with the edited manuscript, he(she) will receive on offprint order form for extra reprints, which will be charged to the authors. 

Rev. bras. oceanogr.. $50($ único): 75.2002

\section{AGRADECIMENTOS AOS ASSESSORES}

Os membros da Comissão Editorial do Instituto Oceanográfico da Universidade de São Paulo agradecem aos especialistas que contribuíram com suas revisões cuidadosas e sugestões valiosas para a qualidade dos trabalhos publicados no volume 50(único) da Revista Brasileira de Oceanografia.

\section{ACKNOWLEDGEMENTS OF REFEREES}

The editors are pleased to record their thanks to all those listed below, who gave their time and expertise to referee papers accepted for publication on the Brazilian Journal of Oceanography. Their contributions have helped ensure the publication of quality science.

Alberto Figueiredo Jr. - Brazil/Brasil

Carmen L. D. B. Rossi-Wongtschowski - Brazil/Brasil

Demetrio Boltovskoy - Argentine/Argentina

Jarbas Bonetti Filho - Brazil/Brasil

Jean-Paul Casanova - France/França

Lauro Júlio Calliari - Brazil/Brasil

Manuel I Iaimovici - Brazil/Brasil

Miguel Petrere $\mathrm{J}_{\mathrm{r}}$. - Brazil/Brasil

Moyses Gonsalez Tessler -- Brazil - Brasil

Richard B. Deriso - United States/Estados Unidos

Tokio Wada - Japan/Japão 
Diagramação e Montagem:

Teresa Cristina dos Reis Gonzalez

Julio Costa Gonzalez

Revisão: Cássia Cerezani de Andrade Oliveira

Difusão: Biblioteca "Prof. Dr. Gelso Vazzoler"

Instituto Oceanográfico da USP Seção de Apoio à Produção Editorial 2002 